\title{
Magnetic Circuit Design for Electromagnetic flow Transducer with Locally Shrunk Measurement Pipe*
}

\author{
Tiejun Liu, Guangming Zhang \\ College of Metrological and Measurement Engineering, China Jiliang University, Hangzhou 310018, China \\ tjliu@cjlu.edu.cn,924161694@qq.com
}

\begin{abstract}
Based on the analysis of the magnetic circuits of the electromagnetic flow transducers with uniform circular pipe, locally shrunk circular pipe and locally shrunk rectangular pipe, the sensitivities of the different designs were compared. The design with locally shrunk rectangular pipe was adopted, the efficiency of the magnetic circuit was effectively enhanced, the sensitivity of the transducer was increased. A prototype flow meter was developed based on the proposed design and was tested. The experiment result shows that the prototype has the precision better than $0.5 \%$ over the specified measurement range. When powered with a high power lithium battery set, the prototype can work for more than 3 years continuously. The feasibility of the proposed design was proved.

Index Terms - Electromagnetic flow meter, Locally shrunk pipe, Velocity distribution, Power consumption
\end{abstract}

\section{Introduction}

Electromagnetic Flow meters are widely used in the measurement of the flow rate of different electrically conductive mediums. Recent research activities in this field are mainly focused on the development of electromagnetic flow meters with very low power consumption, the development of electromagnetic flow meters with extended measurement ability for mediums of very low conductivity and multi-phase medium .Many researchers have made efforts in the study of different technologies to reduce the power consumption of electromagnetic flow meters[1-7].In this paper some new technologies for battery powered electromagnetic flow meters are discussed.

The power for an electromagnetic flow meter is mainly consumed for two purposes, one of them is to produce the magnetic field for the electromagnetic transducer so the Faraday's law can work, the other purpose is to pick up the electrical potential from the electrodes of the transducer and process it to produce a DC signal representing the medium flow rate .The consumption for the first purpose is the power consumed of the magnetic circuit of the transducers, and the consumption for the second purpose is actually the consumption of the conversion circuit .Compared with each other, the power consumption of the magnetic circuit is much larger than that of the conversion circuit. Techniques for low power conversion circuits design have been widely discussed [1-3]. It is more difficult to reduce the power consumption of the magnetic circuit with no loss in the sensitivity of the transducer, for this purpose the structure and construction materials of the transducer must be optimised[8][9].
In this paper, the magnetic circuits of the electromagnetic transducers with different measurement pipe structures are analyzed. The pipe structures considered include the uniform circular pipe, the pipe with locally shrunk circular crosssection and the pipe with locally shrunk rectangular crosssection .The efficiency of the magnetic circuits in different designs are compared .It is proved that the design with a circular pipe locally shrunk to rectangular cross-section provides higher efficiency than the other designs. A prototype flow meter with a locally shrunk rectangular pipe and micropower electronic circuits was designed and manufactured. The flow meter prototype was tested and the proposed design was proved to be feasible.

\section{Transducer with Uniform Circular Pipe}

In an electromagnetic flow meter, electrically conductive fluid flows through a pipe of non-magnetic material submerged in a magnetic field, the inductive potential is picked up with two electrodes at the pipe wall. Practically, the pipe is an uniform circular pipe, the imagined line connecting the two electrodes runs across the central line of the pipe. The magnetic field, the flow direction and the imagined connecting line of electrodes are perpendicular to each other.

Bevir[10] proved that in the case of an uniform magnetic field, the potential on the electrodes depends on the medium velocity distribution in the measurement pipe. The coordination system is defined that the $\mathrm{x}$-axis is located on the line connecting the two electrodes, the y-axis is vertical to the $\mathrm{x}$-axis and points to the direction of the magnetic field, the $\mathrm{z}$ axis located on the central line of the measurement pipe. A weight function $\mathrm{W}_{0}(\mathrm{x}, \mathrm{y}, \mathrm{z})$ can be defined to give the potential contribution by the medium element at $(\mathrm{x}, \mathrm{y}, \mathrm{z})$ running at the velocity of one unit. If the medium flow in the pipe is fully developed as in most cases, the flow velocity will not depend on dimension $\mathrm{z}$. In such case the weight function can be simplified to a two-dimensional scalar function, denoted as $\mathrm{W}(\mathrm{x}, \mathrm{y})$.

$$
W(x, y)=\int_{-\infty}^{+\infty} W_{0}(x, y, z) d z
$$

And the potential between the electrodes can be represented as in

\footnotetext{
${ }^{*}$ This work is supported by the national natural science foundation of China (No.51305419) and the application development program of Chongqing science \& technology commission (No.csts2013yykfC00001).
} 


$$
E_{\mathrm{i}}=\frac{4}{\pi D^{2}} \int_{S} B V(x, y) W(x, y) d x d y,
$$

where $E_{i}$ denotes the potential between the electrodes, $B$ denotes the magnetic flux density, $\mathrm{D}$ denotes the diameter of the pipe, $\mathrm{V}(\mathrm{x}, \mathrm{y})$ denotes the average flow rate of the measured fluid, $\mathrm{S}$ denotes the pipe cross-section on $\mathrm{x}-\mathrm{y}$ surface.

A magnetic transducer according to formula (1) and (2) is not practical. One reason is that an uniform magnetic field in large space is not practical. Another reason is that the required length of the measurement pipe is at least two times of the pipe diameter, this makes a compact flow meter design impossible. And when the potential between the electrodes depends on the velocity distribution, the measurement precision is limited[10] [11].

Practically a pair of excitation coils in the saddle shape are employed in an electromagnetic flow transducer with uniform circular pipe. In such cases, the magnetic field is limited to the space near the pipe cross-section on which the electrodes are located, the required pipe-length is reduced. If magnetic field distribution can be denotes as $\mathrm{B}(\mathrm{x}, \mathrm{y}, \mathrm{z})$, then

$$
B(x, y)=\int_{-\infty}^{+\infty} B(x, y, z) d z .
$$

The excitation coils are specially designed to guarantee

$$
B(x, y) W(x, y)=B_{0} .
$$

If the requirement of formula (4) is fully met, the potential between the electrodes will not depend on the velocity distribution in the uniform circular measurement pipe. The average medium velocity can be represented as

$$
V_{\mathrm{av}}=\frac{4}{\pi D^{2}} \iint V(x, y) d x d y .
$$

Then the potential output between the electrodes can be represented as

$$
E_{i}=B_{0} D V_{a v} .
$$

\section{Transducer with Locally Shrunk Pipe}

It is known from (6) that the potential output of an electromagnetic flow transducer is proportional to the magnetic field strength $\mathrm{B}_{0}$, the distance between the electrodes and the average velocity to be measured. So the sensitivity of the transducer can be enhanced by improving the transducers structure to enhance the magnetic field, to extend the distance between electrodes or to enhance the medium velocity. When a low power design of magnetic transducer is considered, a locally shrunk measurement pipe can be used to get a higher local velocity in the measurement part and the magnetic field can be enhanced without increasing electrical current in the excitation coils as the distance between the coils are shorter than that in the case of an uniform pipe. If the locally shrunk measurement pipe has entrances of internal diameter $\mathrm{D}$, the cross-section area at pipe entrances can be deducted as

$$
S_{0}=\frac{\pi D^{2}}{4} .
$$

The shrunk part of the measurement pipe has a circular or rectangular cross-section whose area S1 is smaller than S0. If the measured medium is taken as incompressible and the average velocity at the pipe entrance is $\mathrm{V}_{\mathrm{av}}$, then the average velocity at the shrunk part will be enhanced to $V_{a v 1}$.

$$
V_{\text {av } 1}=\frac{S_{0}}{S_{1}} V_{a v}
$$

When designing a battery powered flow meter, an important parameter is the ratio between the magnetic flux density B and the electrical current I flowing in the excitation coils .This will be defined as the excitation efficiency of the transducer, specified as in

$$
K=\frac{B}{I}
$$

Where $\mathrm{K}$ denotes the excitation efficiency, $\mathrm{B}$ denotes the average magnetic field strength between coils, I denotes the electrical current flowing in the excitation coils.

A. Measurement Pipe with circular Shrunk part

In the electromagnetic flow transducer, the magnetic field excited by a pair of coils can be represented as in equation (10), (11) and (12).

$$
F=N \cdot I
$$

where $\mathrm{F}$ is the magnetic potential difference between the two coils, I is the exciting current in the coils and $\mathrm{N}$ is the turns of the coil.

$$
R_{m}=\frac{L}{\mu S}
$$

where $\mathrm{Rm}$ is the magnetic resistance, $\mathrm{L}$ is the length of the magnetic circuit, $\mu$ is the magnetic permeability and $\mathrm{S}$ is the cross-section area of the magnetic circuit.

$$
\begin{aligned}
& B=\frac{F}{R_{m} S}=\frac{F \mu}{L}=\frac{N I \mu}{L} \\
& K=\frac{B}{I}=\frac{N \mu}{L}
\end{aligned}
$$

As shown in (13), a power effective transducer design requires that a higher excitation efficiency $\mathrm{K}$ is attained by increasing the turns of the coil and reducing the distance between the two coils.

In the case that the pipe has circular entrances of diameter D and locally shrunk circular part of diameter d, using (7) and (8) the average velocity $V_{\text {avs } 1}$ in the shrunk part can be calculated from the average velocity at the entrance $\mathrm{V}_{\mathrm{av}}$.

$$
V_{\text {avs } 1}=\frac{S_{0}}{S_{1}} V_{a v}=\frac{D^{2}}{d^{2}} V_{a v}
$$


If the distance between the coil and the internal wall of the pipe is hd, and suppose this is valid for the pipes of any diameter, then with the same turns of coil and the same exciting current $I$, the excitation efficiency can be increased by using a shrunk pipe.

$$
\frac{B_{s 1}}{B_{0}}=\frac{K_{s 1} \cdot I}{K_{0} \cdot I}=\frac{N \mu / L_{s 1}}{N \mu / L_{0}}=\frac{D+2 h_{d}}{d+2 h_{d}}
$$

Where $\mathrm{K}_{\mathrm{S} 1}$ is the excitation efficiency of the transducer with a shrunk circular pipe, and $\mathrm{K}_{0}$ is the excitation efficiency of the transducer with an uniform circular pipe of diameter $D$.

Then the electrical potential output of the transducer with a locally shrunk circular pipe can be calculated.

$$
E_{\mathrm{is} 1}=\frac{B_{s 1} d V_{a v s 1}}{B_{0} D V_{a v}} E_{i}=\frac{D\left(D+2 h_{d}\right)}{d\left(d+2 h_{d}\right)} E_{i}
$$

In the case that a measurement pipe with nominal diameter $50 \mathrm{~mm}$, hd is about $7 \mathrm{~mm}$, and suppose $\mathrm{d}$ is $25 \mathrm{~mm}$, the cross-section area at the shrunk part is one 4th of that at the pipe entrance. From (15) and (16) it is easy to know that $\mathrm{K}_{\mathrm{S} 1}$ is about 1.64 times of $\mathrm{K}_{0}$, and $\mathrm{E}_{\mathrm{iS} 1}$ is about 3.28 times of $\mathrm{E}_{\mathrm{i}}$.

\section{B. Measurement Pipe with Shrunk Rectangular Part}

In the case of a transducer with a shrunk rectangular pipe, the cross-section area of the shrunk part is given by

$$
S_{0}=W H
$$

Where $\mathrm{W}$ and $\mathrm{H}$ are respectively the width and the height of the internal cross-section of the pipe at the shrunk part.

Suppose the distance between the coil and the internal wall of the pipe is also $h_{d}$, the turns of the coil is $\mathrm{N}$ and the current in the coil is $\mathrm{I}$ as in the case of an uniform pipe transducer. Then the ratio between the average magnetic flux density at the shrunk rectangular part BS2 and B0 as in the case of an uniform pipe design is as in equation (18).

$$
\frac{B_{s 2}}{B_{0}}=\frac{K_{s 2} \cdot I}{K_{0} \cdot I}=\frac{N \mu / L_{s 2}}{N \mu / L_{0}}=\frac{D+2 h_{d}}{H+2 h_{d}}
$$

Where $\mathrm{K}_{\mathrm{S} 2}$ is the excitation efficiency of the transducer with a shrunk rectangular pipe, and $\mathrm{K}_{0}$ is the excitation efficiency of the transducer with an uniform round pipe.

And the average velocity $\mathrm{V}_{\text {avs2 }}$ in the shrunk part can be calculated from the average velocity at the entrance $V_{a v}$.

$$
V_{\mathrm{avs} 2}=\frac{S_{0}}{S_{2}} V_{a v}=\frac{\pi D^{2} / 4}{W H} V_{a v}=\frac{\pi D^{2}}{4 W H} V_{a v}
$$

Then the electrical potential output of the transducer with a locally shrunk rectangular pipe can be calculated.

$$
E_{\mathrm{is} 2}=\frac{B_{s 2} d V_{a v s 2}}{B_{0} D V_{a v}} E_{i}=\frac{\pi D\left(D+2 h_{d}\right)}{4 H\left(H+2 h_{d}\right)} E_{i}
$$

For a measurement pipe with nominal diameter $50 \mathrm{~mm}, \mathrm{~h}_{\mathrm{d}}$ is about $7 \mathrm{~mm}$. In the transducer design with a rectangular shrunk part, $\mathrm{W}$ is usually not so much less than $\mathrm{D}$, as an example $\mathrm{W}$ can be taken as $0.8 \mathrm{D}$. Suppose the cross-section area of the shrunk rectangular part is also one 4th of that of the pipe entrance, the pressure loss will be roughly the same. The height $\mathrm{H}$ of the shrunk rectangular part can be given by

$$
H=\frac{\pi D^{2} / 4}{4 W}=\frac{\pi D^{2}}{16 \cdot(0.8 D)} \approx 0.245 D .
$$

for convenience $\mathrm{H}$ can be taken as $12 \mathrm{~mm}$, then from (18) it is known that $\mathrm{K}_{\mathrm{S} 2}$ is 2.46 times of $\mathrm{K}_{0}$. and from (20) it can be deducted that $\mathrm{E}_{\mathrm{iS} 2}$ is about 8.0 times of $\mathrm{E}_{\mathrm{i}}$.

When the locally shrunk rectangular pipe is adopted for the transducer design, two rectangular planar electrodes are optimal for picking up the electrical potential. A nearly uniform magnetic field in the vicinity of the electrodes can be obtained from a pair of rectangular coils. From such a electromagnetic flow transducer, the potential between electrodes will not depend on the flow rate distribution in the measurement pipe [10][11].

\section{Transducer Prototype and Experiment Result}

An electromagnetic flow transducer with a locally shrunk rectangular pipe outputs a potential 8.0 times as high as that of a transducer with an uniform circular pipe for the same average medium velocity and with the same exciting current for the magnetic coils, this make it ideal for low power flow meter designs. When matched with planar electrodes and rectangular excitation coils, the potential output of the transducer will not depend on the velocity distribution in the pipe. This makes the transducer design with a locally shrunk rectangular pipe is preferable for power consumption and precision considerations.

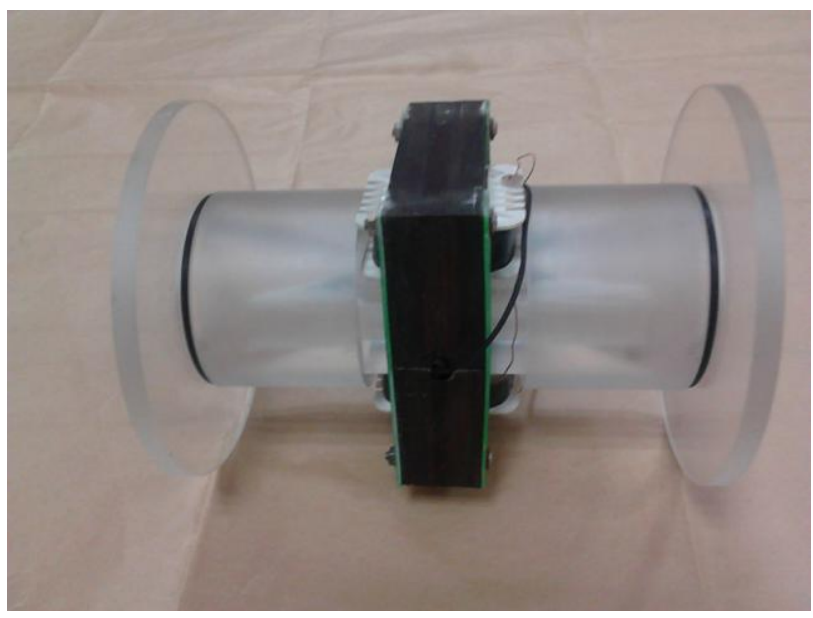

Fig. 1 the Prototype Electromagnetic Flow Meter

As shown in Fig.1, a prototype electromagnetic flow meter was designed in which a transducer with locally shrunk rectangular pipe and micro-power electronic circuits were incorporated .The pipe of the transducer has entrances of $50 \mathrm{~mm}$ diameter and the total length of the pipe is $200 \mathrm{~mm}$. The shrunk part of the pipe has a length of $50 \mathrm{~mm}$ and a rectangular cross-section of $15 \mathrm{~mm}$ in height and $45 \mathrm{~mm}$ in 
width. A pair of rectangular coils are used for producing a nearly uniform magnetic field in the vicinity of the electrodes. The electrodes are rectangular, having the dimensions of $14 \mathrm{~mm}$ in height and $20 \mathrm{~mm}$ in length.

The micro-power conversion circuit consume an electrical current of $10 \mathrm{~mA}$ in active mode and $20 \mathrm{uA}$ in static mode. One measurement is taken per 3 seconds, the measurement lasts $50 \mathrm{~ms}$ in which the coils are excited with an electrical current of $50 \mathrm{~mA}$. The average current consumed by the flow meter is

$$
I=[(50+10) \times 50] / 3000+0.02=1.02 \mathrm{~mA} .
$$

The energy consumed in one year will be

$$
E=1.02 \times 24 \times 30 \times 12=88128 m A h .
$$

When powered by a battery set of 4 lithium batteries and each battery has energy storage of $8500 \mathrm{mAh}$, the prototype can work continuously for 3 years .

The prototype flow meter was tested with tap water on a calibration system using weighing method. The precision of the calibration system is $0.1 \%$. The test result of the prototype is shown in Table I. The precision of the prototype flow meter is better that $0.5 \%$.

\begin{tabular}{|c|c|c|c|c|c|}
\hline$\%$ & $\begin{array}{c}\text { Flow rate } \\
{[\mathrm{m} 3 / \mathrm{h}]}\end{array}$ & $\begin{array}{l}\text { Volume } \\
\text { [Litre ] }\end{array}$ & $\begin{array}{c}\text { Reading } \\
\text { [Litre] }\end{array}$ & $\begin{array}{c}\text { Error } \\
\text { [Litre] }\end{array}$ & $\begin{array}{c}\text { Relative } \\
\text { Error[\%] }\end{array}$ \\
\hline 100 & 35 & $\begin{array}{l}555.004 \\
557.809 \\
560.683\end{array}$ & $\begin{array}{l}552.9 \\
555.5 \\
558.6\end{array}$ & $\begin{array}{l}-2.104 \\
-2.309 \\
-2.083\end{array}$ & $\begin{array}{l}-0.38 \\
-0.41 \\
-0.37\end{array}$ \\
\hline 50 & 17.5 & $\begin{array}{l}314.400 \\
308.797 \\
310.351\end{array}$ & $\begin{array}{l}313.5 \\
308.4 \\
309.2\end{array}$ & $\begin{array}{c}-0.9 \\
-0.397 \\
-1.151\end{array}$ & $\begin{array}{l}-0.29 \\
-0.13 \\
-0.37\end{array}$ \\
\hline 20 & 7 & $\begin{array}{l}110.415 \\
110.218 \\
111.634\end{array}$ & $\begin{array}{l}110.8 \\
109.9 \\
111.3\end{array}$ & $\begin{array}{c}0.385 \\
-0.318 \\
-0.334\end{array}$ & $\begin{array}{c}0.35 \\
-0.29 \\
-0.30\end{array}$ \\
\hline 10 & 0.7 & $\begin{array}{l}108.062 \\
107.259 \\
108.872\end{array}$ & $\begin{array}{l}107.8 \\
106.9 \\
108.6\end{array}$ & $\begin{array}{l}-0.262 \\
-0.359 \\
-0.272\end{array}$ & $\begin{array}{l}-0.24 \\
-0.33 \\
-0.25\end{array}$ \\
\hline
\end{tabular}

TABLE I Prototype Test Result

\section{Conclusions}

Compared with the traditional electromagnetic flow transducer design with an uniform pipe, an electromagnetic flow transducer with locally shrunk rectangular pipe has the advantages of high sensitivity, less power consumption. When a pair of rectangular coils are designed for producing the magnetic field and rectangular planar electrodes are adopted for picking up the potential, the potential output of the proposed transducer design has minimum dependence on the velocity distribution in the pipe, high precision measurement of the velocity can be realized.

An electromagnetic flow transducer with locally shrunk rectangular pipe was designed and made, micro-power electronic circuits were incorporated for a prototype flow meter. The prototype flow meter was tested and proved to have the precision better than $0.5 \%$.Powered with a battery set of 6 Lithium batteries, the prototype flow meter can continuously work more than 3 years.

\section{References}

[1] R. Katutis, R. Vaikasas, and J. Virbalis, "The Battery- Driven Electromagnetic Flow Converter with Reduced Power Consumption," Electronics and Electrical Engineering, no.1, pp.53-56, 2010.

[2] R.Padegimas, J. Virbalis, and R. Vaikasas, "Selection of the Magnetic Circuit Design for Electromagnetic Fluid Flow Converter with Rectangular Channel", Electronics and Electrical Engineering, no.3,pp.41-46,2006.

[3] Xiaoyu Jin, Zhaotang Su, and Deju Mo, "Design of the measurement circuit of low power electromagnetic flowmeter". Chinese journal of Electronic instrumentation Customer, no.1,pp.23-25,2005.

[4] Tiejun Liu, Tongsheng Gong, and YinjiaChen, "Study on low power electromagnetic flow meter with locally shrunk measurement pipe", Chinese Journal of Sensor and Actuators, vol.26,no.3,pp.348352, march 2013

[5] Changqi Li, Yaxian Zhang, and Limei Sun, "Magnetic Field excitation technique in Battery Powered Electromagnetic Flow meter", Chinese Journal of Digital Technology and Application,no.8,pp.11-12,2009.

[6] Kaixia Wei, Bin Li, and Jianzhen Chen, "Study on the method of liquid surface measurement in an Electromagnetic flow meter", Chinese Journal of Sensors and Actuators, vol.21, no.12, pp.2106-2110, December 2008.

[7] Bin Li, Jun Yao, and Xia Li, "The Analysis and Application of the Rectangular Electromagnetic Flow meter", Proceedings of IMTC2003Instru- mentation and Measurement Technology Conference, pp.490494, Vail Co USA, 20-23 May 2003.

[8] Jiao Huang, Chun Yao, and Ting Ding, "Design of an Electromagnetic flow meter based on new excitation method", Chinese Journal of Sensors and Actuators, vol.23, no.2, pp.215-219, February 2010.

[9] JA. Shercliff, The Theory of Electro-magnetic Flow Measurement, $1^{\text {st }}$ ed., London, Cambridge University Press, 1962, pp.1-65.

[10] BEVIR MK. "The theory of induced voltage electromagnetic flow meter “, Fluid Mechanics, vol.43, no.3, pp.577-590, march 1970.

[11] CC. SMYTH, "Derivation of weight functions for the circular and rectangular channel magnetic flow meters by means of Green's theorem and conformal mapping", Physics E: Scientific, Instrument,no.4,pp.4752,1970 . 